\title{
Results of the implementation of work on the creation of virtual test benches for testing the digital twin of the bus
}

\author{
Andrey Skripko ${ }^{1}$, Elena Zhilenkova ${ }^{1}$ and Anton Zhilenkov ${ }^{2, *}$ \\ ${ }^{1}$ Peter the Great St. Petersburg Polytechnic University, Institute for Advanced Manufacturing \\ Technologies, Center of National Technological Initiative, 195251, St. Petersburg, Russian \\ Federation \\ ${ }^{2}$ Saint Petersburg State Marine Technical University, Cyber-Physical Systems Department, 190121, \\ St. Petersburg, Russian Federation
}

\begin{abstract}
The results of the implementation of work in the field of creating virtual test benches for testing digital twins of products in the automotive industry are presented. The results of the implementation of virtual benches for assessing the water tightness and drainage of bus bodies are specifically considered. The structure of the virtual bench for assessing the sound absorption of materials in the alpha chamber is given. The results of the development of the concept of a virtual test bench for the bus electrical equipment simulation are presented.
\end{abstract}

\section{Introduction}

Digital twin technologies are increasingly being used both in design and testing systems for specific complex technical objects, and in general in various industries. They are the basis of virtual benches, on which virtual tests of various properties and characteristics of complex technical objects and their individual elements are carried out.

The possibility of conducting virtual tests, in turn, makes it possible to carry out activities for the certification of objects. A complex of virtual benches can form a so-called digital platform.

For such complex technical objects as a bus, a digital platform for their design and testing should include a number of virtual benches and corresponding digital models. It should make it possible to assess passive safety and the operation of certain systems, such as, for example, an electrical system, a power plant, etc.

Moreover, such a platform should allow assessing the sound insulation of the structure, the water resistance, the quality of the drainage system, etc. At the early stages of design, it is necessary to first create parametric computational models and the corresponding automated software systems.

\footnotetext{
*Corresponding author: zhilenkovanton@gmail.com
} 


\section{General view of the digital platform}

The described digital platform was developed for the design and testing of commercial and passenger vehicles, including passenger buses. The digital platform of a passenger bus should include a number of specific test benches and corresponding digital twins. To date, a digital platform has been developed and it includes the following:

- Bench 1D for modeling the temperature in the engine cooling system;

- Bench for assessing the temperature regime in the engine shaft of the buses;

- Bench for assessing the fuel consumption of city buses;

- Bench for assessing the indicators of passive safety of a bus during rollover according to UN-ECE Regulation No. 66;

- Bench for assessing the fouling of the outer panels of the bus exterior;

- Bench for assessing the noise in the passenger compartment of the bus, given the interior design and forces acting from the suspension;

- Bench for assessing water permeability and drainage of bus body;

- Bench for assessing the sound absorption of materials in the alpha chamber;

Thus, the platform allowed SPbPU engineers to develop a modular roof design concept for a number of buses. Moreover, SPbPU engineers have developed a concept of a virtual test bench for modeling the electrical equipment of a modern bus.

\section{Virtual benches based on the digital model of the bus}

The primary task of designing a passenger or commercial vehicle is to ensure the safety of its operation. Thus, the main test and subject of certification is the assessment of the vehicle passive safety during various collisions with other objects. This is an assessment of the Apillars impact, frontal impact, overhead or rear impact, as well as a passive safety rating in the event of a side impact. Figure 1. gives an overall view of some results obtained at the virtual bench for assessing the passive safety during crash test.

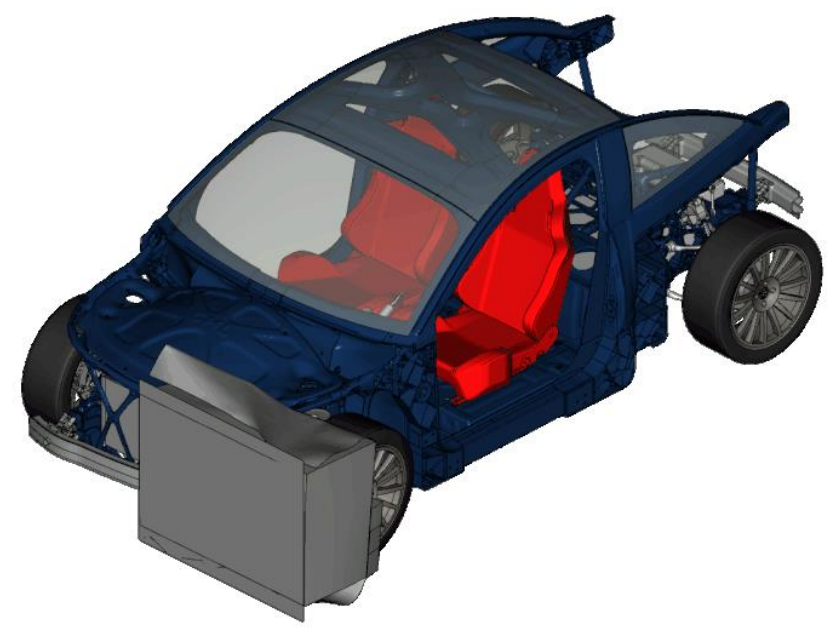

Fig. 1. Some examples of visualization of the results obtained at the virtual bench for assessing vehicle passive safety.

It is obvious that modeling such effects requires an appropriate mathematical model or a digital twin. The results obtained depend on the selected parameters of the vehicle body structure, its pillars, chassis, etc. Moreover, the results of the assessment can be also used to optimize and improve the design parameters in automatic mode. 
Equally important for urban transport is the exterior of the vehicle. It refers not just to the aesthetic aspects of the appearance of the designed product, but to the fouling of the exterior. Figure 2-3 shows some types of user interface that reflect the capabilities of the digital bench. Figure 2 shows the output of the system for assessing the level of fouling. The designer or product examiner can assess not only the main fouling areas while the vehicle is in motion, but also the intensity of fouling in various parts of the exterior.
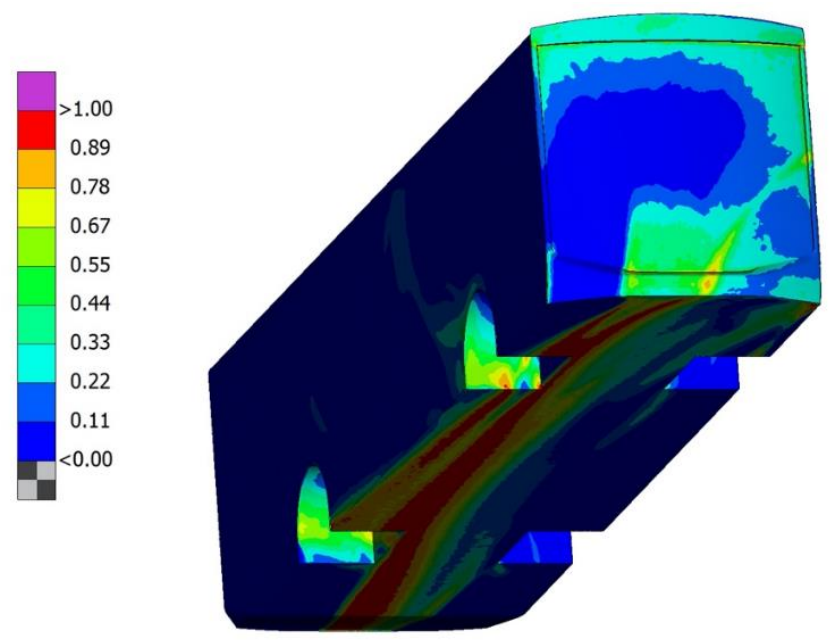

Fig. 2. The result of simulation of the degree of fouling of the outer surface of the bus.

Figure 3 shows a screenshot of a part of the interface, which reflects the result of modeling an incoming flow that spreads dirt over the exterior area as the vehicle moves. It can be seen that the spread of dirt depends on the shape of the body, the presence of curvature of decorative and structural elements, etc.

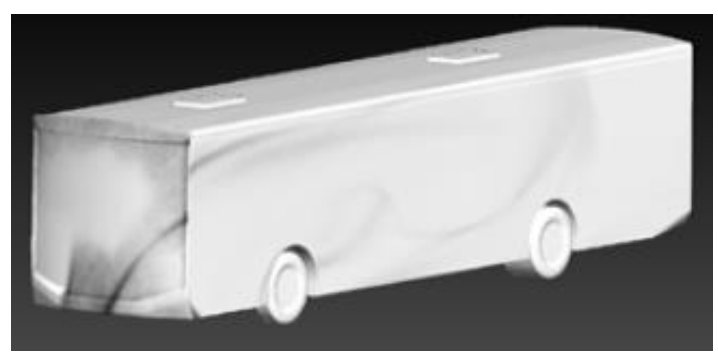

Fig. 3. Modeling the spread of dirt of the bus exterior by the incident flow.

Simulation based on the digital twin makes it possible to assess the noise in the bus interior, given its peculiarities. This virtual bench allows not only assessing the level of inside noise coming from outside, but also the noise from the suspension. In order to do this, a model that calculates the forces acting on the bus body from the side of the suspension and their contribution to the noise observed inside the bus is implemented in the digital twin. 

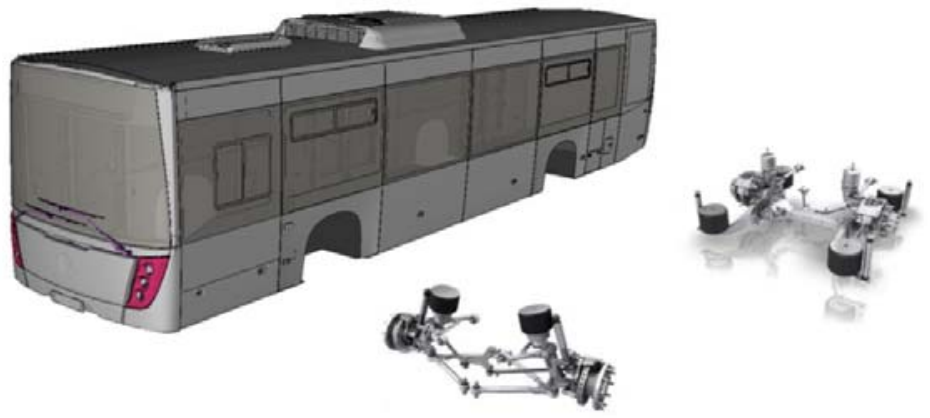

Fig. 4. Virtual bench for assessing acoustic noise inside a vehicle.

Figure 5 shows the frequency response, which makes it possible to estimate the noise level inside the bus at a particular frequency.

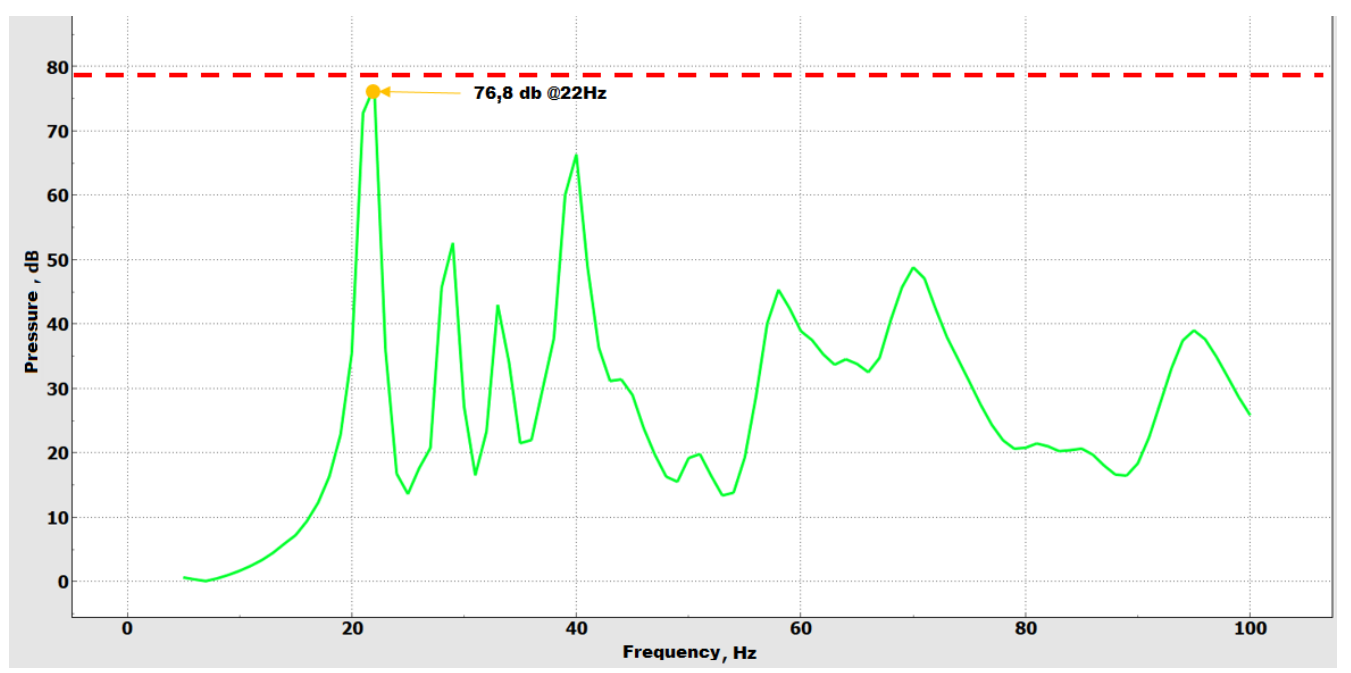

Fig. 5. Element of the user interface, which shows the amplitude-frequency characteristic of the noise inside the bus, obtained as an assessment on the virtual bench of the digital platform.

The last type of testing and assessment presented in this article is the testing of water resistance and drainage of the vehicle body. Figure 6 is a screenshot of a user interface element showing a bus under the influence of the water flows. 


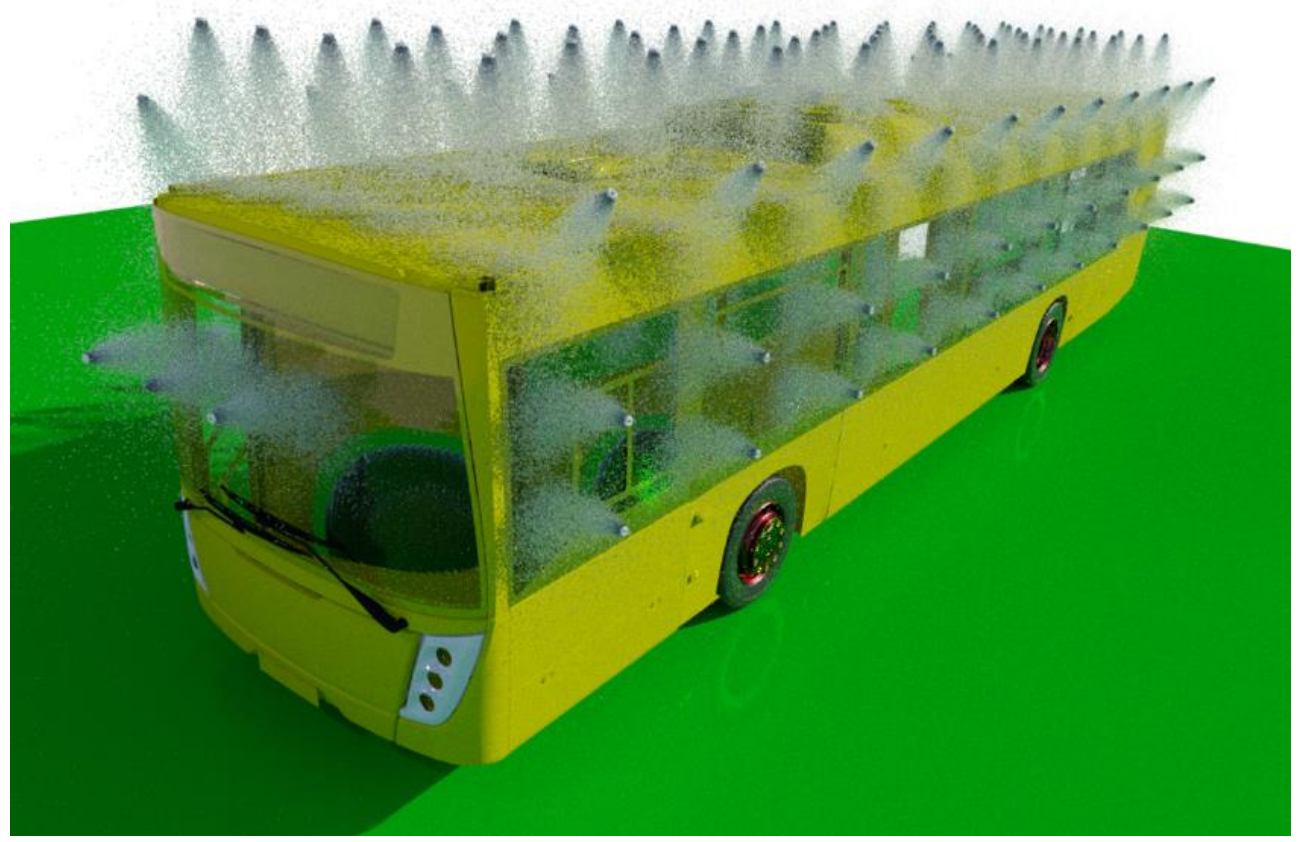

Fig. 6. Element of the graphical user interface of the virtual test bench for water protection and drainage of the vehicle body.

Figure 7 shows the results of testing the properties of water drainage of the water flow acting on the bus body and windows. This test, implemented on the basis of a digital twin, makes it possible to assess the quality of visibility of the situation on the road from the driver's seat at different intensity and direction of the influencing water flow.
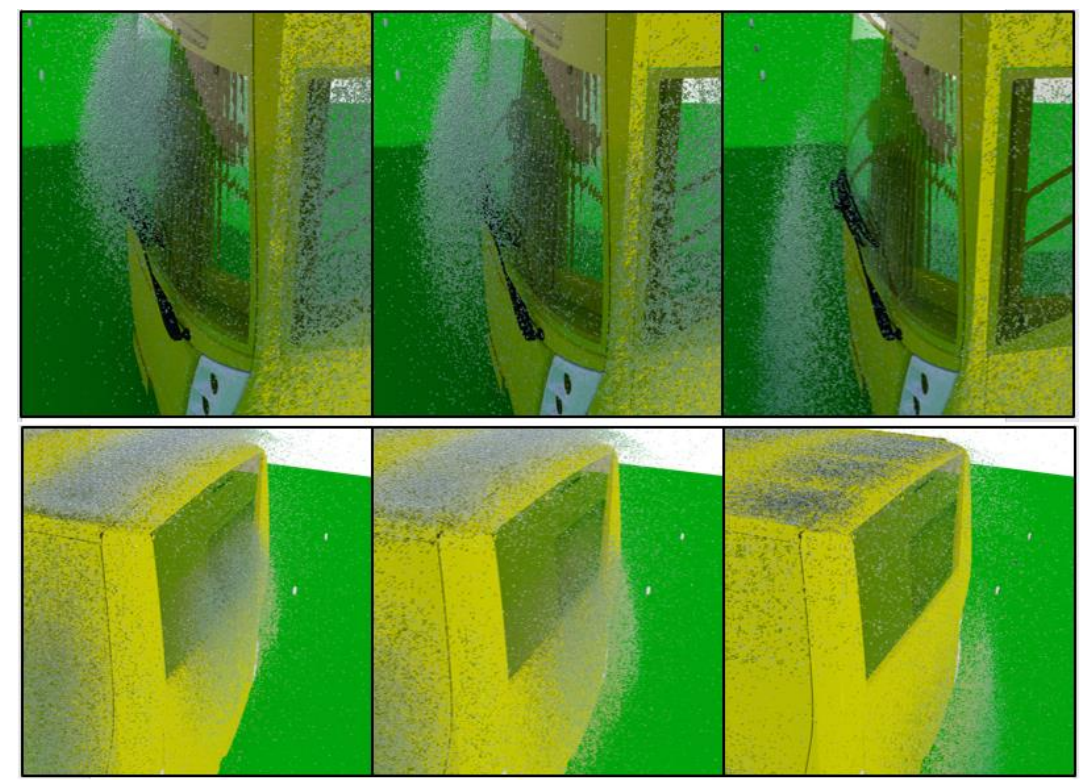

Fig. 7. Examples of simulation obtained on a virtual bench for testing the drainage properties of the body of a designed vehicle. 
The drainage from the bus roof for various configurations and flow rates is also shown.

\section{Conclusion}

The results obtained in this article show that digital platforms allow solving tasks that are difficult or impossible to solve on full-scale models, or that are much more expensive and time-consuming. Modern technologies in the field of digital platforms for the design and testing of complex technical products provide methods and tools for in-depth analysis of the most diverse aspects of the designed systems operation. Thus, the presented virtual bench for assessing the vehicle passive safety provides the ability to simulate a variety of extreme situations and assess their consequences for the vehicle and, especially, for the people in it. The relation of the test data to the design features of the body, the properties of the materials used makes it possible to analyze and detect the features and limitations of design objects, which were difficult or impossible to detect on existing prototypes.

Unique tests on the level of acoustic noise inside the vehicle, including noise from the suspension elements, have been demonstrated. It is not mentioned in the article, but to date a virtual bench simulating tests in an alpha chamber has already been implemented. The unique model, which describes the noise in the cabin from the suspension, also makes it possible to evaluate and optimize the influence of the suspension, its elements and materials on the noise level inside the vehicle body. Such assessments provide an opportunity to analyze the properties of acoustic noise inside the body depending on the frequency range. Therefore, it allows optimizing the materials used in the design of the vehicle interior, soundproofing materials, etc.

The virtual bench for assessing the fouling of the vehicle exterior thus implements a virtual bench on which the incident flow around the body of the designed product is simulated. Carrying out additional calculations and analysis, it became possible to assess the fouling of the exterior and the influence of the body shape on it, even to minor protrusions on the surface, decorative curves and functional irregularities.

Last but not least, a virtual bench for analyzing the water protection of the body, as well as for assessing its properties to ensure water drainage, was demonstrated. As has been shown, it is extremely important for assessing visibility from the driver's seat when a vehicle moves in a water flow, which is created by rain, water masses on the road, etc.

One of the most important problems of the potential or already realized possibilities of virtual benches and digital platforms remains the problem of ensuring the adequacy of the mathematical model of a digital twin, on which benches and platforms are created. It requires the solution of different problems. These are the problems of obtaining analytical solutions to various kinds of problems, and ensuring the convergence and stability of calculations in the intervals of the target modes of their operation. The latter leads to the problem of providing sufficient computing power, which is also directly related to the problem of analytical solutions or the need to use purely computational schemes, or their combinations. The implementation of numerical methods results in the need to solve problems of optimization of parallel computations, computations on GPUs and other similar problems related to hardware solutions.

Thus, constantly developing, digital twin technology still requires solving the full range of problems typical for simulation systems. It includes theoretical and fundamental problems, as well as problems of hardware implementations. Moreover, the accuracy of the solutions they provide must constantly increase, and the relationship between fundamental problems and hardware is becoming increasingly stronger.

\section{References}


1. D. Lisitsa, A.A. Zhilenkov, IEEE Conf. of Russian Young Researchers in Electrical and Electronic Engineering (EIConRus) 922 (2017) doi: 10.1109/EIConRus.2017.7910707

2. A.D. Karpov, A.A. Zhilenkov, D. Lisitsa, IEEE Conference of Russian Young Researchers in Electrical and Electronic Engineering (EIConRus) 887 (2017) doi: 10.1109/EIConRus.2017.7910697

3. V.N. Kozlovsky et al., IOP Conf. Ser.: Mater. Sci. Eng. 986, 012047 (2020)

4. R. Petrov, P. Cvetkov, A. Maksimov, O. Klyavin, Int. J. Mech 14, 119 (2020)

5. A.A. Zhilenkov, D. Denk, IEEE Conference of Russian Young Researchers in Electrical and Electronic Engineering (EIConRus) $\mathbf{1 1 0 0}$ (2017) doi: 10.1109/EIConRus.2017.7910747

6. A.V. Ivanov, A.A. Zhilenkov, IEEE Conference of Russian Young Researchers in Electrical and Electronic Engineering (EIConRus) $\mathbf{8 8 2}$ (2018) doi: 10.1109/EIConRus.2018.8317229

7. F. Jaensch, A. Csiszar, C. Scheifele, A. Verl, 25th International Conference on Mechatronics and Machine Vision in Practice (M2VIP). IEEE 11 (2018)

8. Q. Gemine, D. Ernst, B. Cornélusse, Optim Eng. 18, 587 (2017)

9. A.V. Ivanov, A.A. Zhilenkov, IEEE Conference of Russian Young Researchers in Electrical and Electronic Engineering (EIConRus) 890 (2018) doi: 10.1109/EIConRus.2018.8317231 